\title{
Abdominal Wall Endometrioma Mimicking Pubalgia: A Case Report
}

\author{
Thiago Sampaio Busato ${ }^{1}$, Juliana Maranho ${ }^{2}$, William Kondo ${ }^{3}$, Marcelo Morozowski ${ }^{4}$, Gladyston \\ R Matioski Fo ${ }^{4}$, Lucas Dias Godoi ${ }^{4}$ and Juan R V Capriotti ${ }^{5}$ \\ ${ }^{1}$ Chair of Hip Surgery Fellowship Programme, Brazil
}

${ }^{2}$ Fellow of Hip Surgery Fellowship Programme, Brazil

${ }^{3}$ Gynecologist of VITA Batel and Hospital das Nações, Brazil

${ }^{4}$ Staff of Hip Surgery Unit at C.R.I.Ar, Brazil

${ }^{5}$ Chair of Hip Surgery Unit at C.R.I.Ar, Brazil

*Corresponding author: Thiago S Busato, Chair of Hip Surgery Fellowship Programme, Brazil

ARTICLE INFO

Received: 鏗 July 11, 2020

Published: 幽 July 24, 2020

Citation: Thiago Sampaio B, Juliana M, William K, Marcelo M, Gladyston R Matioski F, et al., Abdominal Wall Endometrioma Mimicking Pubalgia: A Case Report. Biomed J Sci \& Tech Res 29(1)-2020. BJSTR. MS.ID.004752.

Keywords: Pubis; Endometrioma
ABSTRACT

The authors present a case of endometrioma of the rectus abdominis muscle that mimicked a clinical condition of pubalgia in a female patient. There was a previous history of two cesarean deliveries and an abdominoplasty. The patient underwent prior unsuccessful physiotherapy for pubalgia in another service until an MRI scan finally clarified the correct etiological diagnosis. The initial pelvic radiographs presented, curiously, an image compatible with the pubic symphysis's osteoarthritis, probably inducing the initial diagnosis of the assistant colleague for a mechanical etiology, given that the patient was also quite active. After the new diagnosis, we referred the patient to a gynecological surgery team specialized in the treatment of endometriosis. The patient achieved complete resolution of symptoms after tumor resection, which was histologically compatible with an endometrioma. Endometriosis of the rectus abdominis muscle, as described in this clinical case, is rare, with about only 20 cases found in the literature. In the knowledge of the authors, this is it's first report as a differential diagnosis of pubalgia. When it forms a circumscribed mass, endometriosis is called an endometrioma. One of the most common sites for the appearance of endometrioma is in the lower abdominal wall, especially if there is a local scar (by cesarean section or other surgery). This case report calls for rare but relevant differential diagnoses of pubalgia. It should always be considered in women with suspicious symptoms and a history of previous abdominal surgery, especially cesarean. For the definitive diagnosis, in addition to complete semiology, a radiological investigation that includes nuclear magnetic resonance is routinely necessary.

\section{Introduction}

Pubalgia is a painful inflammatory syndrome of variable etiology that involves the pubic symphysis and its neighboring structures [1]. Among the main factors involved in its pathogenesis, sports that involve microtrauma in the pubic region stand out. Less often, it is secondary to infectious causes or gynecological and urological surgery [2]. The clinical condition starts with pain in the pubic region. It can occur irradiation to the perineum and the inner thigh region and worsens on exertion $[1,3,4]$. It is usually a self- limited condition, but in athletes who maintain physical activity in the presence of the disease, the pain can worsen, becoming limiting $[1,2]$. The diagnosis is initially clinical, but should always be based on imaging tests. Irritating maneuvers (squeeze test, Grava test) can cause pain. Imaging exams may show mechanical overload in the region, for example, due to the presence of pubic symphysis arthrosis on radiographs and T2 bone edema on Magnetic Resonance Imaging (MRI), in addition to possible tendonitis of the 
adductor muscles or abdominal rectum. The treatment is initially conservative, with physiotherapy, rest, and anti-inflammatory drugs. On the rare occasions when the painful condition persists even after conservative treatment, surgical treatment, indicated on a case-by-case basis, may be required, including debridement of the pubic symphysis, partial pubectomy, adductor, and rectus abdominis tenotomy, or arthrodesis with use bone graft [5]. There is a multitude of differential diagnoses for pubalgia. Among them are intra-articular pathologies of the hip, urological diseases, abdominal wall dysfunctions, and gynecological diseases [6-9]. We highlighted the abdominal wall endometrioma, with few reports of occurrence in the world literature [8-10]. In the previous literature, we did not find a description of an abdominal wall endometrioma mimicking a pubalgia condition, as described in this report. The patient fully consented that her medical data and clinical (and radiological) images would be used for this case report. The authors declare that there are no conflicts of interest in this report.

\section{Case Report}

39-year-old female patient. Housewife, a practitioner of fitness and weight training in the gym. She sought our service for a second opinion with a previous diagnosis of pubalgia, as reported.
Complained of chronic pain in the pubic region for approximately a year, with gradual worsening. She reported pain worsening after physical exercise and at night. When questioned, she reported cyclic worsening related to menstrual cycles and history of two cesarean deliveries and an abdominoplasty. She also reported temporary pain improvement after the intramuscular application of corticosteroids, relapsing days later. She had been followed up in another service and physiotherapy for six months, without favorable results. Physical examination showed a normal gait. A typical transverse scar from an abdominoplasty in the lower abdomen was noted. Upon palpation, there was pain in the rectus abdominis muscle topography at the right side of the lower abdominal region (without a palpable mass). The range of motion of the hips was normal. There was no crural pain, the femoroacetabular impingement maneuvers were negative, as well as the irritative sacroiliac maneuvers. The squeeze test was negative, and although she presented mild pain to Grava's test, she was still able to flex the trunk. She had a radiograph in anteroposterior view of the pelvis that showed discrete osteoarthritis of the pubic symphysis and bilateral morphology of pincer-type acetabular impingement (asymptomatic) (Figure 1).

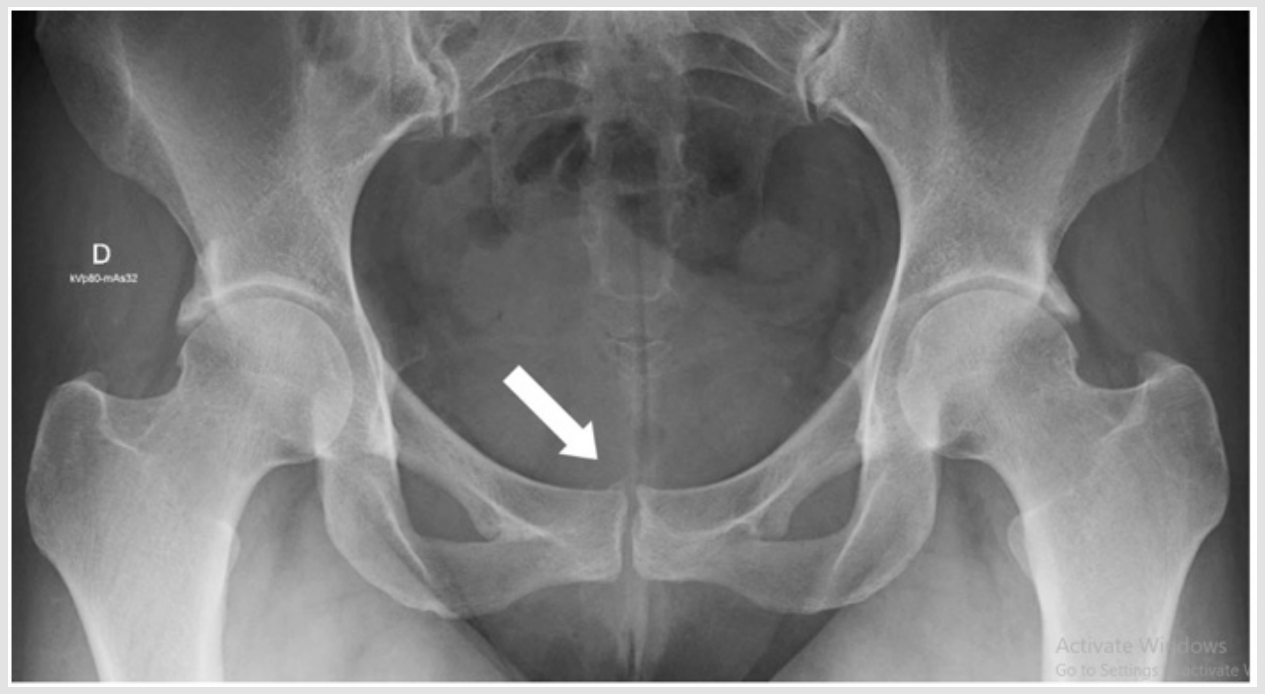

Figure 1: Radiograph of the pelvis in anteroposterior view. White arrow demonstrates osteoarthritis of the pubic symphysis.

New radiographs were requested, including the "flamingo" series for pubalgia, and a MRI. These showed the presence of a Morfological pincer-type impingement and confirmed the previous findings of pubic symphysis arthrosis, in addition to mild sclerosis of the sacroiliac. The flamingo series did not present significant unevenness to alternating monopodial support. MRI of the pelvis demonstrated an oval lesion, with a fusiform aspect, poorly delimited, predominantly hypointense in $\mathrm{T} 1$ and with an intermediate signal in T2 / STIR, measuring about $40 \mathrm{~mm}$ in the longest axis of the muscle right abdominal rectum, about $12 \mathrm{~mm}$ from the insertion in the pub, with diffuse enhancement by the contrast. There was no tendonitis or bone edema. Therefore, abdominal wall endometriosis was suspected (Figures 2 \& 3). Based on the imaging findings and the endometrioma suspect, the patient was referred to a gynecological team specialized in endometriosis. Surgical removal of the lesion was indicated. For this, the surgeon made a $5 \mathrm{~cm}$ incision on the previous scar, dissecting the planes up to the fascia of the rectus abdominis muscle. The lesion was just cranial to the pubic symphysis and was removed entirely. A nodule with a typically endometriotic aspect was observed (Figures 4 \& 5). The patient evolved uneventfully in the postoperative period, with total improvement in pain symptoms at 4 weeks after surgery. The anatomopathological examination confirmed the initial hypothesis of endometrioma. After a year of follow-up, the patient remains asymptomatic and has resumed her physical activities. 


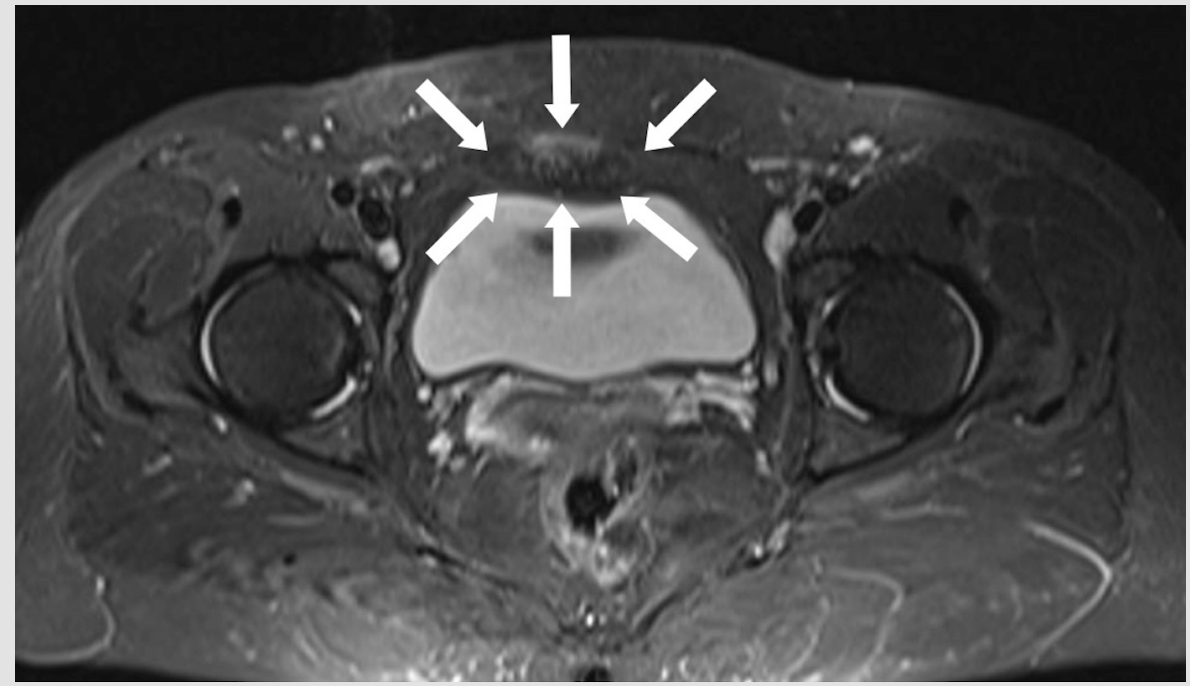

Figure 2: Magnetic Resonance Imaging of the pelvis, axial section. White arrows demonstrate the tumor.

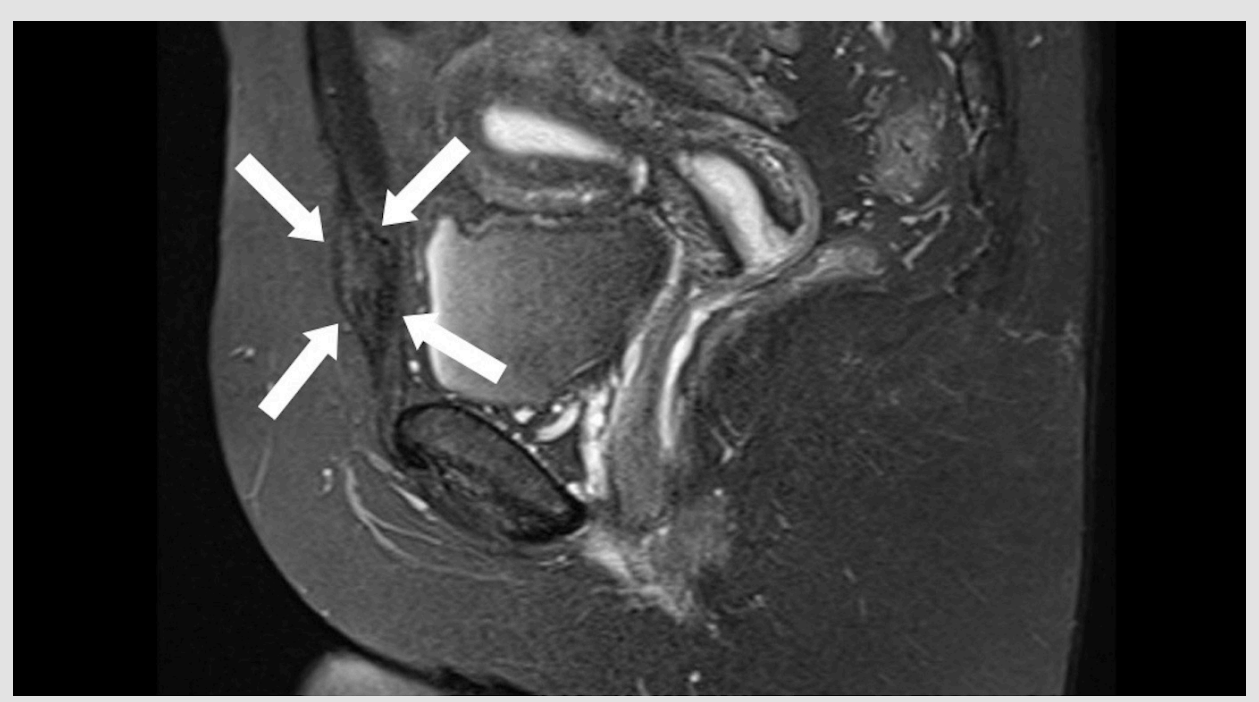

Figure 3: Magnetic Resonance Imaging of the pelvis, sagittal section. White arrows demonstrate the tumor.

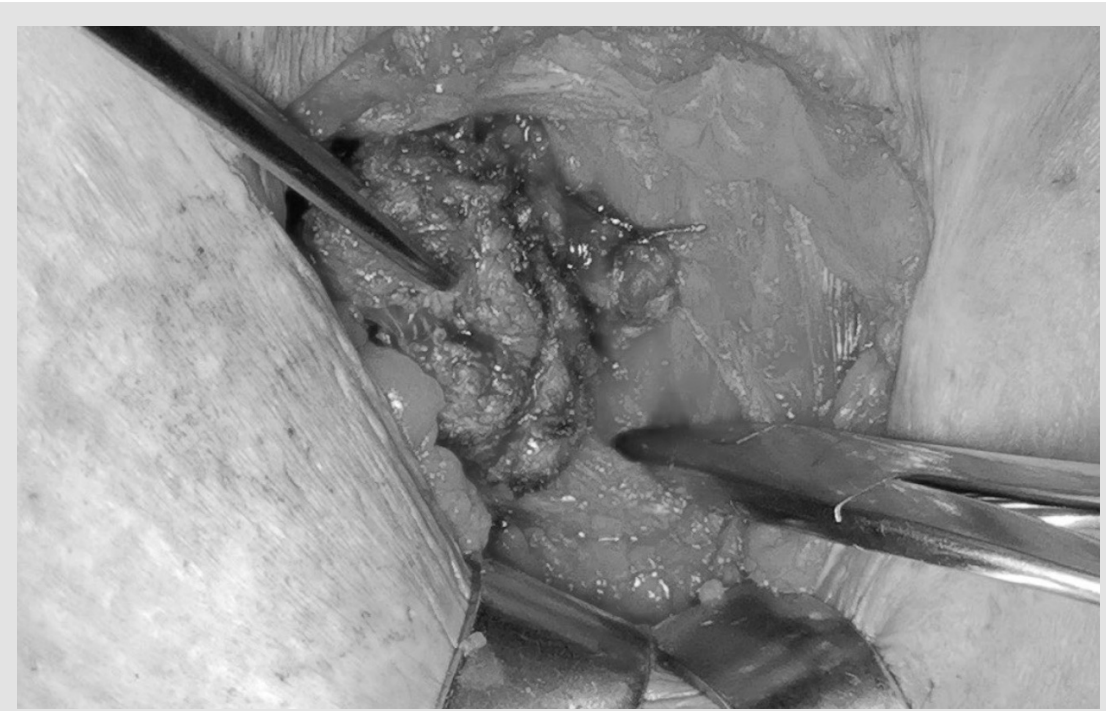

Figure 4: Intraoperative image. The tumor is dissected from the right rectus abdominis muscle belly. 


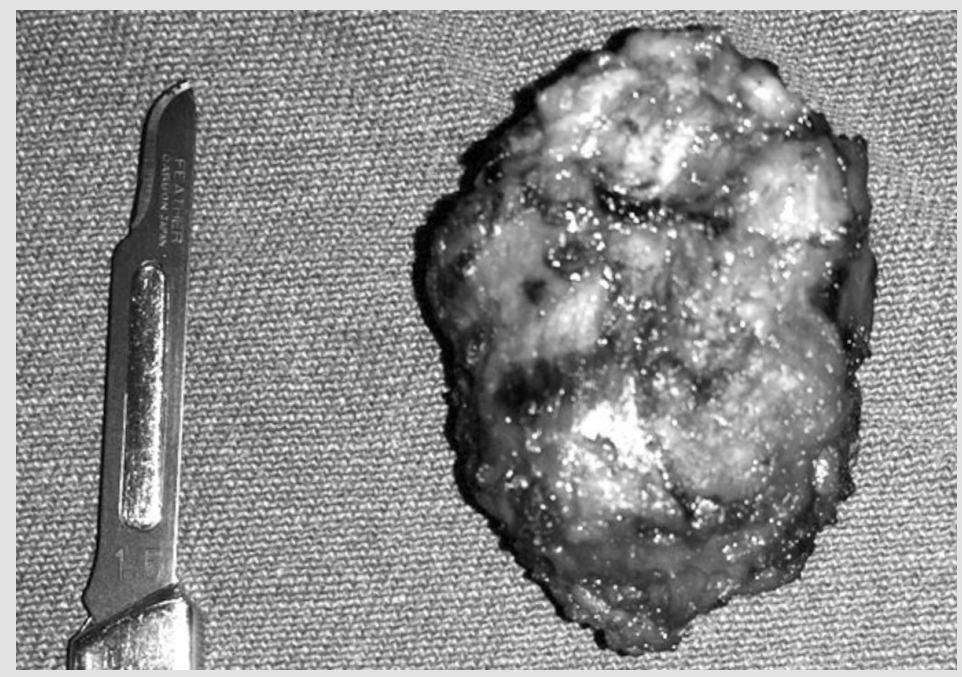

Figure 5: Image of the specimen (\#15 scalpel blade for reference). Source: authors' files.

\section{Discussion}

Several terms are used as synonyms for pubalgia, such as pubic osteitis (used when there is an inflammatory cause), athlete's pubalgia, among others [4]. It is common to complain about the presence of pain in the pubic symphysis region, which can irradiate to the abdomen, perineum, and inner thigh. Pain tipically progressively worsens with movement and improves with rest [2,3,4]. Treatment is eminently non-surgical, with physiotherapy, withdrawal from sports, systemic or local corticosteroids, and non-hormonal anti-inflammatory drugs. Among the differential diagnoses of pubalgia are intra-articular pathologies of the hip, sacroiliitis, urological diseases, inguinal hernias, and gynecological diseases [6-9]. In this last group, there are ovarian cysts and abdominal wall endometriosis. Endometriosis is defined as the presence of tissue from the endometrium located outside the uterus. The prevalence ranges from 8 to $15 \%$ of women of childbearing age 10 and can be either pelvic or extra-pelvic. Endometriosis of the rectus abdominis muscle, as described in this clinical case, is rare, with only about 20 cases found in the literature [8-10]. When it forms a circumscribed mass, endometriosis is called an endometrioma [8]. One of the most common sites for the appearance of endometrioma is in the lower abdominal wall, especially if there is a local scar (by cesarean section or other surgery). The incidence of abdominal wall endometrioma after the cesarean section varies from 0.03 to $0.8 \%$ of cases $[8,9]$. Although the number of women affected by pubalgia is still lower than in men, the incidence tends to increase, with the increasing participation of women in high-impact sports related to the genesis of the disease (running, football, hockey, etc.). One of the factors cited as causative of the lower incidence of pubalgia in women could be the shape of the female pelvis. The gynaecoid pelvis has a larger area of insertion of the abdominal muscles, which increases the area of distribution of forces and could prevent the disease [9]. Even so, it is necessary to value situations that can confuse the diagnosis of pubalgia in women. This is due to a large number of differential diagnoses, such as gynecological pathologies, which can mimic the symptoms of the disease in the case presented here.

In this report, a patient with chronic pain was shown to have a failure similar to that of her pubic pain. This case was first diagnosed as pubalgia of mechanical etiology due to the location of the pain and presenting local alteration in the pubic symphysis on the radiograph, which was compatible with the condition initially suspected by the other colleagues. Besides, the presence of femoroacetabular impingement-although asymptomatic in this case - could also lead to diagnostic error. However, the failure in the previous treatment and the incomplete radiological investigation led us to consider other differential diagnoses. In this case, previous gynecological surgeries and localized pain in the abdominal wall's musculature alerted us to the possibility of some local alteration. MRI is essential as a diagnostic tool in the suspicion of pubalgia, being considered a gold-standard diagnostic test [1]. Therefore, we must pay attention to the possibility of gynecological etiology in patients who present symptoms of pubalgia, but mainly in those who are not part of the most familiar profile to the disease or who do not respond adequately to the initial treatment.

\section{Conclusion}

This case report calls attention for a rare but important differential diagnosis of pubalgia. It should always be considered in women with suspicious symptoms and a history of previous abdominal surgery, especially cesarean. For the definitive diagnosis, in addition to the complete semiology, a radiological investigation that includes nuclear magnetic resonance is routinely necessary.

\section{References}

1. Gomes LSM (2015) Cirurgia Preservadora do Quadril Adulto. Atheneu pp. 359-361. 
2. Grava de Sousa JP, Fallopa F, Siqueira Jr D, Santa Cruz ARS (2005) Tratamento cirurgico da pubalgia em jogadores de futebol profissional. Rev Bras Ortop 40(10): 601-607.

3. Schuroff A, Pedroni M, Deeke M, Valerio J, Vargas M, et al. (2012) Pubalgia como uma das causas de dispareunia. Rev Port Ortop Traum 20(1).

4. Reis FA, Rosenfeld A, Ikawa MH, Silva FD, Costa JD, et al. (2008) A importância dos exames de imagem no diagnóstico da pubalgia no atleta. Rev Bras Reumatol 48(4).

5. Matikainen M, Hermunen H, Paajanen H (2017) Athletic pubalgia in females: predictive value of MRI in outcomes of endoscopic surgery. Orthop J Sports Med 5(8): 2325967117720171.

6. Oliveira AL, Andreoli CV, Ejnisman B, Queiroz RD, Pires OGN, et al (2016) Perfil epidemiológico dos pacientes com diagnóstico de pubalgia do atleta. Rev bras ortop 51(6).

ISSN: $2574-1241$

DOI: $10.26717 /$ BJSTR.2020.29.004752

Thiago Sampaio Busato. Biomed J Sci \& Tech Res

(C) This work is licensed under Creative

Submission Link: https://biomedres.us/submit-manuscript.php
7. Branco RC, Fontenelle CRC, Miranda LM, Ching San Jr YA, Vianna EM (2010) Estudo comparativo por ressonância magnética de púbis entre atletas e sedentários assintomáticos. Rev bras ortop 45(6).

8. Ozkan OF, Cikman O, Kiraz HA, Roach EC, Karacaer MA, et al. (2014) Endometrioma localized in the rectus abdominis muscle: A case report and review of literature. ABCD arq bras cir dig 27(3).

9. Accetta I, Accetta P, Accetta AF, Maia FJS, Oliveira APFA (2011) Endometrioma de parede abdominal. ABCD arq bras cir dig 24(1).

10. Gachabayov M, Horta R, Afanasyev D, Gilyazov T (2016) Abdominal wall endometrioma: Our experience in Vladimir, Russia. Niger Med J 57(6): 329-333.

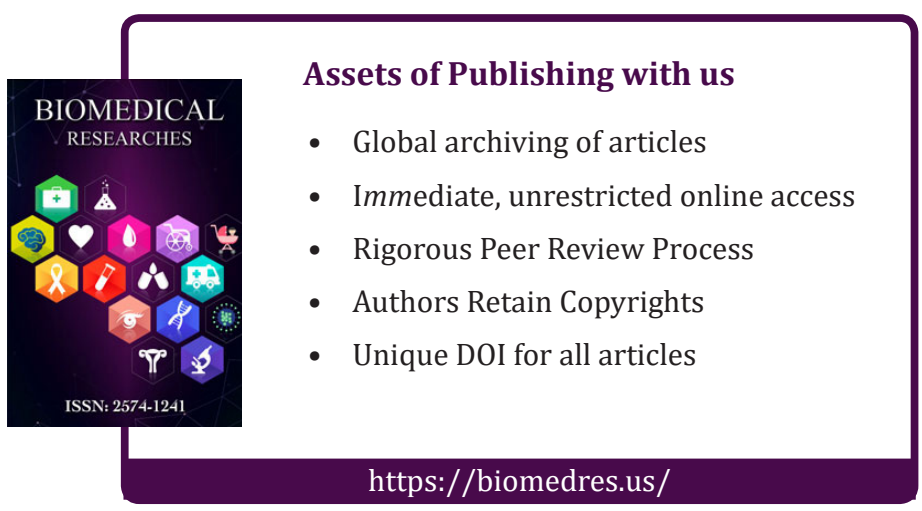

